\title{
Generalized Sphere-Packing Bound for Subblock-Constrained Codes
}

\author{
Han Mao Kiah ${ }^{\dagger}$, Anshoo Tandon*, and Mehul Motani* \\ $\dagger$ School of Physical and Mathematical Sciences, Nanyang Technological University, Singapore \\ * Electrical \& Computer Engineering, National University of Singapore \\ Emails: hmkiah@ntu.edu.sg, anshoo.tandon@gmail.com, motani@nus.edu.sg
}

\begin{abstract}
We apply the generalized sphere-packing bound to two classes of subblock-constrained codes. À la Fazeli et al. (2015), we made use of automorphism to significantly reduce the number of variables in the associated linear programming problem. In particular, we study binary constant subblock-composition codes (CSCCs), characterized by the property that the number of ones in each subblock is constant, and binary subblock energy-constrained codes (SECCs), characterized by the property that the number of ones in each subblock exceeds a certain threshold. For CSCCs, we show that the optimization problem is equivalent to finding the minimum of $N$ variables, where $N$ is independent of the number of subblocks. We then provide closed-form solutions for the generalized spherepacking bounds for single- and double-error correcting CSCCs. For SECCs, we provide closed-form solutions for the generalized sphere-packing bounds for single errors in certain special cases. We also obtain improved bounds on the optimal asymptotic rate for CSCCs and SECCs, and provide numerical examples to highlight the improvement.
\end{abstract}

\section{INTRODUCTION}

Subblock-constrained codes are a class of constrained codes where each codeword is divided into smaller subblocks, and each subblock satisfies a certain application-dependent constraint. Subblock-constrained codes have recently gained attention as they ' are suitable candidates for applications such as simultaneous energy and information transfer [1], visible light communication [2], low-cost authentication methods [3], and powerline communications [4].

In this paper, we discuss two important subclasses of subblock-constrained codes. The first subclass are the constant subblockcomposition codes (CSCCs). Binary CSCCs have varied applications [2], [3], [5], and are characterized by the property that 'each subblock in every codeword has the same weight, i.e. each subblock has the same number of ones. The second subclass of subblock-constrained codes that we study are the subblock energy-constrained codes (SECCs) which ensure that the energy content in every subblock of each codeword exceeds a certain threshold [1]. SECCs have application in simultaneous energy and information transfer [1], and binary SECCs are characterized by the property that the number of ones in each subblock is at least $w$. Bounds on the capacity and error exponent for SECCs and CSCCs over noisy channels were presented in [1], while bounds on the SECC and CSCC code size and asymptotic rate, with minimum distance constraint, were analyzed in [6].

In this paper, we study a modified version of the generalized sphere-packing bound à la Fazelli et al. [7]. A specialized version of the generalized bound was first introduced by Kulkarni and Kiyavash [8] in the context of deletion-correcting code and since then, variants of their method were applied to a myriad of coding problems (see [7] for a survey). Fazeli et al. then studied their method in a general setup and provided what is called the generalized sphere-packing bound. Now, the generalized sphere-packing 'bound is essentially given by the optimal solution of a linear programming problem and in most cases, determining its exact value is difficult. Nevertheless, we apply the symmetry techniques in [7] to significantly reduce the size of the linear program and our main contributions are the closed-form solutions of the generalized sphere-packing bound in certain cases for CSCCs and SECCs.

We also extend the results in [6] to present improved upper bounds on the asymptotic rates for CSCCs and SECCs for a range of relative distance values. These results are obtained by applying a generalized version of the sphere-packing bound (Sec. III), and by judiciously choosing appropriate constrained spaces for estimating asymptotic ball sizes.

\section{Generalized SPHere-PACKING Bound}

We give a modified version of the sphere-packing bound in full generality, and then specialize it to the class of codes that we are interested in.

Let $\tau$ be a distance metric defined over $\Sigma^{n}$ and pick some constrained space $\mathcal{S} \subseteq \Sigma^{n}$. A subset $\mathcal{C} \subseteq \mathcal{S}$ is an $(n, d ; \mathcal{S})$-code if $d=$ $\min \{\tau(\boldsymbol{x}, \boldsymbol{y}): \boldsymbol{x}, \boldsymbol{y} \in \mathcal{C}, \boldsymbol{x} \neq \boldsymbol{y}\}$ and we are interested in determining the value $A(n, d ; \mathcal{S}) \triangleq \max \{|\mathcal{C}|: \mathcal{C}$ is an $(n, d ; \mathcal{S})$-code $\}$.

Fix $d$ and set $t=\lfloor(d-1) / 2\rfloor$. For $\boldsymbol{x} \in \mathcal{S}$, let $\mathcal{B}(\boldsymbol{x}, t)$ be the ball $\left\{\boldsymbol{y} \in \Sigma^{n}: \tau(\boldsymbol{x}, \boldsymbol{y}) \leq t\right\}$. We further define $\mathcal{T} \triangleq \bigcup_{\boldsymbol{x} \in \mathcal{S}} \mathcal{B}(\boldsymbol{x}, t)$. In other words, $\mathcal{T}$ is the set of all words whose distance is at most $t$ from some word in $\mathcal{S}$.

We consider a binary matrix $M$ whose rows are indexed by $\mathcal{S}$ and columns are indexed by $\mathcal{T}$. Set

$$
\boldsymbol{M}_{\boldsymbol{x}, \boldsymbol{y}}= \begin{cases}1 & \text { if } \tau(\boldsymbol{x}, \boldsymbol{y}) \leq t \\ 0 & \text { otherwise }\end{cases}
$$


Theorem 1 (Fazeli et al. [7], Cullina and Kiyavash [9]). For $d \leq n$, set $t$ and $M$ as above. Then

$$
A(n, d ; \mathcal{S}) \leq \min \left\{\sum_{\boldsymbol{y} \in \mathcal{T}} Y_{\boldsymbol{y}}: \boldsymbol{M Y} \geq \mathbf{1}, Y_{\boldsymbol{y}} \geq 0 \text { for } \boldsymbol{y} \in \mathcal{T}\right\} .
$$

Usually, we consider spaces $\mathcal{S}$ whose size is exponential in $n$. Therefore, determining the exact value of (1) by solving the linear program directly is computationally prohibitive. Hence, most authors [7], [9] chose certain feasible points in the linear program (1) and evaluated the objective functions to obtain upper bounds on $A(n, d ; \mathcal{S})$. In particular, Cullina and Kiyavash [9] introduced the local degree iterative algorithm to pick "good" feasible points. In the following theorem, we picked feasible points by judiciously choosing constrained spaces that contain $\mathcal{S}$ and estimating the corresponding ball sizes. This is motivated by Freiman's and Berger's methods [10], [11] that improve the usual sphere-packing bounds for constant weight codes.

Formally, we choose $\tilde{\mathcal{S}} \subseteq \Sigma^{n}$, a subset possibly different from $\mathcal{S}$. For $\boldsymbol{x} \in \mathcal{S}$, define $\mathcal{B}_{\tilde{\mathcal{S}}}(\boldsymbol{x}, t) \triangleq\{\boldsymbol{y} \in \tilde{\mathcal{S}}: \tau(\boldsymbol{x}, \boldsymbol{y}) \leq t\}$ and set $V_{\mathcal{S}, \tilde{\mathcal{S}}}^{\min }(t) \triangleq \min \left\{\left|\mathcal{B}_{\tilde{\mathcal{S}}}(\boldsymbol{x}, t)\right|: \boldsymbol{x} \in \mathcal{S}\right\}$.

Theorem 2. Set $t=\lfloor(d-1) / 2\rfloor$. For any $\tilde{\mathcal{S}} \subseteq \Sigma^{n}$, if $V_{\mathcal{S}, \tilde{\mathcal{S}}}^{\min }(t) \geq 1$, then

$$
A(n, d ; \mathcal{S}) \leq \frac{|\tilde{\mathcal{S}}|}{V_{\mathcal{S}, \tilde{\mathcal{S}}}^{\min _{1}(t)}}
$$

Proof: Abbreviate $V_{\mathcal{S}, \tilde{\mathcal{S}}}^{\min }(t)$ with $V$ and consider the vector $\boldsymbol{Y}$ with entries $Y_{\boldsymbol{y}}$ defined as

$$
Y_{\boldsymbol{y}}= \begin{cases}1 / V & \text { if } \boldsymbol{y} \in \tilde{\mathcal{S}} \\ 0 & \text { otherwise }\end{cases}
$$

We show that vector $\boldsymbol{Y}$ above is a feasible point in the optimization program (1). In other words, we claim that $\boldsymbol{M} \boldsymbol{Y} \geq \mathbf{1}$. Indeed, for $\boldsymbol{x} \in \mathcal{S}$, let $\boldsymbol{M}_{\boldsymbol{x}}$ denote the row of $\boldsymbol{M}$ that corresponds to $\boldsymbol{x}$. We have that

$$
\boldsymbol{M}_{\boldsymbol{x}} \boldsymbol{Y}=\left|\mathcal{B}_{\tilde{\mathcal{S}} \cap \mathcal{T}}(\boldsymbol{x}, t)\right| / V=\left|\mathcal{B}_{\tilde{\mathcal{S}}}(\boldsymbol{x}, t)\right| / V \geq 1
$$

since $V$ corresponds to the smallest ball volume. To complete the proof, it remains to compute the objective value that is $\sum_{\boldsymbol{y} \in \mathcal{T}} Y_{\boldsymbol{y}}=$ $\sum_{\boldsymbol{y} \in \tilde{\mathcal{S}} \cap \mathcal{T}} Y_{\boldsymbol{y}} \leq|\tilde{\mathcal{S}}| / V$.

Observe that there are exponentially many choices for the constrained space $\tilde{\mathcal{S}}$. Nevertheless, in this paper, for the class of CSCCs, we provide a small family of constrained spaces and show that the optimal solution to (1) must be correspond to one of these constrained spaces. Furthermore, the number of these constrained spaces depends only on $t$ and is independent of the length $n$.

Another approach to make the linear program (1) tractable exploits symmetries in the linear program. The approach essentially uses the symmetric structure of the linear program (1) to define another linear program that has significantly lesser variables and constraints, while ensuring the solution to the latter program is the same as the former. The method is formally summarised in Theorem 3 and we remark that similar methods can be found in linear programming literature (see Margot [12], and Bődi and Herr [13]). Independently, Fazelli et al. obtained Theorem 3 in the specialized setting of finding a fractional transversal in hypergraphs and applied it to various coding problems. Here, we describe the method in the language of metric spaces.

Recall that $\tau$ is a distance metric defined over $\Sigma^{n}$. We say that the permutations $\pi: \Sigma^{n} \rightarrow \Sigma^{n}$ is an automorphism with respect to $\tau$ if for all $\boldsymbol{x}, \boldsymbol{y} \in \Sigma^{n}$, we have that $\tau(\boldsymbol{x}, \boldsymbol{y})=\tau(\pi(\boldsymbol{x}), \pi(\boldsymbol{y}))$. It is known that the set $G$ of all automorphisms with respect to $\tau$ form a subgroup of the symmetric group on the set $\Sigma^{n}$.

Consider $X \subset \Sigma^{n}$. A subgroup $H$ of $G$ is $H$-closed if $\pi(\boldsymbol{x}) \in \mathcal{X}$ for all $\pi \in H$ and $\boldsymbol{x} \in \mathcal{X}$. Recall that $\mathcal{T}$ is subset of $\Sigma^{n}$ defined by the constrained space $\mathcal{S}$ and radius $t$. Suppose that $\mathcal{T}$ is $H$-closed and let $O_{1}, O_{2}, \ldots, O_{N}$ be the orbits under the group action of $H$.

Theorem 3 (Fazeli et al. [7]). Given $n, d$, $\mathcal{S}$, we define $t, \mathcal{T}$ and $O_{1}, O_{2}, \ldots, O_{N}$ as above. Then

$$
A(n, d ; \mathcal{S}) \leq \min \left\{\sum_{j \in[N]} Y_{j}^{*}: \boldsymbol{M}^{*} \boldsymbol{Y}^{*} \geq \mathbf{1}, Y_{j}^{*} \geq 0 \text { for } j \in[N]\right\} \text {. }
$$

where $M^{*}$ is a matrix whose rows are indexed by $\mathcal{S}$, columns are indexed by $[N]$, and its entries are given by

$$
\boldsymbol{M}^{*}(\boldsymbol{x}, j)=\sum_{\boldsymbol{y} \in O_{j}} \boldsymbol{M}(\boldsymbol{x}, \boldsymbol{y}) \text { for all } \boldsymbol{x} \in \mathcal{S}, j \in[N] .
$$

Note that $\boldsymbol{M}^{*}(\boldsymbol{x}, j)$ is also the size of the ball $\mathcal{B}_{O_{j}}(\boldsymbol{x}, t)$. 


\section{A. Subblock-Constrained Codes}

In this paper, we focus on the Hamming metric and the following two constrained spaces. Our constrained spaces are parameterized by integers $m, L$ and $w$ with $L / 2 \leq w \leq L$. We consider words of $n \triangleq m L$, where each word is partitioned into $m$ subblocks, each of length $L$. The constant subblock-composition codes (CSCC) space is the space of all words that have constant weight $w$ in each subblock and is denoted by $\mathrm{C}(m, L, w)$. On the other hand, the subblock energy-constrained codes (SECC) space is the space of all words that have weight at least $w$ in each subblock and is denoted by $\mathcal{S}(m, L, w)$. The quantities of interest are hence

$$
\begin{gathered}
C(m, L, d, w) \triangleq A(m L, d ; \mathcal{C}(m, L, w)), \\
S(m, L, d, w) \triangleq A(m L, d ; \mathcal{S}(m, L, w)) .
\end{gathered}
$$

Our contributions are as follow:

(A) We provide exact solutions to the optimization problem given by (1).

- For CSCCs, we show that (1) can computed by finding the minimum amongst a set of at most $N(t)$ values. We demonstrate that $N(t)$ is independent of $m$ and $L$ and provide a combinatorial interpretation of this value in Section III. Furthermore, each of these $N(t)$ values corresponds to choice of constrained space $\tilde{\mathcal{S}}$ in Theorem 2. Using this fact, we provide closed-form solutions for the case $t \in\{1,2\}$.

- For SECCs, we show that (1) can computed by solving a linear program in at most $L^{m} N(t)$ variables. For $t=1$, we provide closed-form solutions for (1) in the following cases: (i) when $m=1$ and $w \geq L / 2$; (ii) when $w=L-1$ and $L \geq m / 2$.

(B) When $d$, or equivalently, $t$, grows linearly with $n$, the reduced optimization problem remains intractable. Nevertheless, we provide estimates to the optimization problem by judiciously choosing appropriate constrained spaces for estimating asymptotic ball sizes and subsquently, applying the generalized sphere-packing bound. Doing so, we extend the results in [6] to present improved upper bounds on the asymptotic rates for CSCCs and SECCs for a range of relative distance values.

\section{Closed-Form Solutions for (1) For Subblock-Constrained Codes}

For words of length $m L$, we consider the following subgroup of automorphisms on $\{0,1\}^{m L}$ with respect to the Hamming metric. Let $H$ be the set of automorphisms that permute the $m$ subblocks and then permute the $L$ coordinates within each block. Let $H$ act on the words $\{0,1\}^{m L}$. Then under this group action, we can index each orbit with an $m$-tuple in

$$
\mathcal{P}(m, L) \triangleq\left\{\left[u_{1}, u_{2}, \ldots, u_{m}\right]: u_{1} \geq \cdots \geq u_{m}, 0 \leq u_{i} \leq L\right\} .
$$

In other words, if we pick a word in $O_{\left[u_{1}, u_{2}, \ldots, u_{m}\right]},\left[u_{1}, u_{2}, \ldots, u_{m}\right]$ is the $m$-tuple obtained by taking the $m$ weights of subblocks and arranging them in non-increasing order.

Example 1. Consider $m=2$ and $L=2$. Under the group action of $H$, the orbits are

$$
\begin{aligned}
O_{[0,0]} & =\{0000\}, \\
O_{[1,0]} & =\{0001,0010,0100,1000\}, \\
O_{[1,1]} & =\{0101,0110,1001,1010\}, \\
O_{[2,0]} & =\{0011,1100\}, \\
O_{[2,1]} & =\{1101,1110,0111,1011\}, \\
O_{[2,2]} & =\{1111\} .
\end{aligned}
$$

Notice that

$$
\begin{aligned}
& \mathcal{C}(m, L, w)=O_{[w, w, \ldots, w]}, \\
& \mathcal{S}(m, L, w)=\bigcup_{u_{1} \geq u_{2} \geq \cdots \geq u_{m} \geq w} O_{\left[u_{1}, u_{2}, \ldots, u_{m}\right]}
\end{aligned}
$$

Therefore, both $\mathcal{C}(m, L, w)$ and $\mathcal{S}(m, L, w)$ are $H$-closed.

To apply Theorem 3, we have to compute the orbit sizes and determine the number of orbits under the action of $H$.

First, we determine the number of words in the orbit corresponding to some $m$-tuple $\boldsymbol{u}=\left[u_{1}, u_{2}, \ldots, u_{m}\right]$. To do so, we introduce the following notation for a multinomial. Let $\boldsymbol{\mu}=\left(\mu_{1}, \mu_{2}, \ldots, \mu_{\ell}\right)$ be a vector of length $\ell$ such that $\sum_{i=1}^{\ell} \mu_{i}=m$. We write

$$
\left(\begin{array}{c}
m \\
\boldsymbol{\mu}
\end{array}\right) \triangleq\left(\begin{array}{c}
m \\
\mu_{1}, \mu_{2}, \ldots, \mu_{\ell}
\end{array}\right)=\frac{m !}{\mu_{1} ! \mu_{2} ! \cdots \mu_{\ell} !}
$$

For $\boldsymbol{u} \in \mathcal{P}(m, L)$, set

$$
N(\boldsymbol{u}) \triangleq\left(\begin{array}{l}
m \\
\boldsymbol{\mu}
\end{array}\right) \text {, where } \mu_{j}=\#\left\{i: u_{i}=j\right\} \text { for } 0 \leq j \leq L \text {. }
$$


Then the size of $O_{u}$ is given by

$$
\left|O_{\boldsymbol{u}}\right|=N(\boldsymbol{u}) \prod_{i=1}^{m}\left(\begin{array}{c}
L \\
u_{i}
\end{array}\right) .
$$

To determine the number of orbits, we look at the respective classes of subblock-constrained codes.

\section{A. Constant Subblock-Composition Codes}

First, we consider the space $\mathcal{S}=\mathcal{C}(m, L, w)$ and set $t=\lfloor(d-1) / 2\rfloor$. Let $\mathcal{T}$ be the resulting space and our task is to determine the number of orbits that are contained in $\mathcal{T}$. Now, the orbits in $\mathcal{T}$ are indexed by the $m$-tuples in the set $\mathcal{P}(m, L ; w, t) \triangleq\{\boldsymbol{u} \in$ $\left.\mathcal{P}(m, L): \sum_{i=1}^{m}\left|u_{i}-w\right| \leq t\right\}$.

Hence, our task is to determine the size of $\mathcal{P}(m, L ; w, t)$. The next proposition states that this number is upper bounded by a value independent of $m, L$, and $w$. Its proof is combinatorial in nature and we defer it to Appendix $\mathrm{A}$

Proposition 4. For all $m, L, w, t$, we have $|\mathcal{P}(m, L ; w, t)| \leq N(t)$, where

$$
N(t)=\sum_{r=0}^{t} \sum_{i=0}^{r} p(i) p(r-i)
$$

and $p(i)$ is the partition number of $i$. Furthermore, we have equality, or $|\mathcal{P}(m, L ; w, t)|=N(t)$, whenever $m \geq t$ and $t \leq$ $\min \{w, L-w\}$.

Next, we set $\boldsymbol{w} \triangleq[w, w, \ldots, w]$ and observe that $\mathcal{C}(m, L, w)=O_{\boldsymbol{w}}$. Applying Theorem 3 , we reduce (1) to the following optimization program.

$$
\min \left\{\sum_{\boldsymbol{u} \in \mathcal{P}(m, L ; w, t)}\left|O_{\boldsymbol{u}}\right| Y_{\boldsymbol{u}}^{*}: \boldsymbol{M}^{*} \boldsymbol{Y}^{*} \geq \mathbf{1}, Y_{\boldsymbol{u}} \geq 0\right\},
$$

$\boldsymbol{M}^{*}$ is the matrix indexed by $\{\boldsymbol{w}\} \times \mathcal{P}(m, L ; w, t)$ whose entries are given by

$$
\boldsymbol{M}_{\boldsymbol{w}, \boldsymbol{u}}^{*} \triangleq\left|B_{O_{u}}\left(\left(0^{L-w} 1^{w}\right)^{m}, t\right)\right| .
$$

Example 2. Consider $m=3, L=10, w=5$ and $t=2$. Then

$$
\begin{aligned}
\mathcal{P}(m, L ; w, t) & =\mathcal{P}(3,10 ; 5,2) \\
& =\{[5,5,5],[6,5,5],[5,5,4],[7,5,5],[6,6,5],[6,5,4],[5,4,4],[5,5,3]\} .
\end{aligned}
$$

Then $M^{*}$ is given by

$$
\boldsymbol{M}^{*}=\left(\begin{array}{llllllll}
76 & 15 & 15 & 30 & 75 & 150 & 75 & 30
\end{array}\right),
$$

and the objective function is given by

$$
\begin{aligned}
& 252^{3} Y_{555}+3(210)(252)^{2} Y_{655}+3(210)(252)^{2} Y_{554} \\
& +3(120)(252)^{2} Y_{755}+3(252)(210)^{2} Y_{665}+6(252)(210)^{2} Y_{654} \\
& +3(252)(210)^{2} Y_{544}+3(120)(252)^{2} Y_{553}
\end{aligned}
$$

Notice that there are eight variables and the feasible region is described by nine constraints (including the eight nonnegative constraints). Hence, each vertex of the feasible region has exactly seven zero components. Therefore, the optimal value of the linear program is given by

$$
\begin{aligned}
\min & \left\{\frac{252^{3}}{76}, \frac{3(210)(252)^{2}}{15}, \frac{3(210)(252)^{2}}{15}, \frac{3(120)(252)^{2}}{30}, \frac{3(252)(210)^{2}}{75}, \frac{6(252)(210)^{2}}{150}, \frac{3(252)(210)^{2}}{75}, \frac{3(120)(252)^{2}}{30}\right\} \\
& =210565.894 .
\end{aligned}
$$

More generally, we provide a closed formula for the exact solution to (5), or equivalently (1).

Proposition 5. For all $m, L, w, t$, the exact solution to (1) for CSCCs is given by

$$
\min \left\{\frac{\left|O_{\boldsymbol{u}}\right|}{\boldsymbol{M}_{\boldsymbol{w}, \boldsymbol{u}}^{*}}: \boldsymbol{u} \in \mathcal{P}(m, L ; w, t)\right\} .
$$

Furthermore, since $|\mathcal{P}(m, L ; w, t)| \leq N(t)$, the solution 6 can be computed in time independent of $m$, $L$, and $w$.

\section{Remark 1.}

(i) For $\boldsymbol{u} \in \mathcal{P}(m, L ; w, t)$, if we set the space $\tilde{\mathcal{S}}$ to be $O_{\boldsymbol{u}}$ in Theorem 2, equation (2) yields the value $\left|O_{\boldsymbol{u}}\right| / \boldsymbol{M}_{\boldsymbol{w}, \boldsymbol{u}}^{*}$. In other words, in order to obtain the best upper bound for Theorem 2 it suffices to consider $\tilde{\mathcal{S}}$ in the collection $\mathbb{S} \triangleq\left\{O_{\boldsymbol{u}}: \boldsymbol{u} \in \mathcal{P}(m, L ; w, t)\right\}$. 
(ii) When $t$ is proportional to $n$, the collection $\mathbb{S}$ is exponential in $n$ and hence, it remains computationally prohibitive to check through all possible constrained spaces. Cullina and Kiyavash [9] introduced the local degree iterative algorithm to iteratively improve the objective value of (1) by suitable modifying the current feasible point. Unfortunately, the algorithm fails to improve all feasible points that correspond to spaces in $\mathbb{S}$.

To conclude this subsection, we apply Proposition 5 to the case $t \in\{1,2\}$.

\section{Corollary 6.}

(i) When $t=1$ and $1 \leq w \leq L-1$, we have that

$$
C(m, L, 4, w) \leq \begin{cases}\frac{\left(\begin{array}{c}
L \\
w-1
\end{array}\right)\left(\begin{array}{l}
L \\
w
\end{array}\right)^{m-1}}{w}, & \text { if } w \leq L / 2, \\
\frac{\left(\begin{array}{c}
L \\
w+1
\end{array}\right)\left(\begin{array}{c}
L \\
w
\end{array}\right)^{m-1}}{L-w}, & \text { otherwise. }\end{cases}
$$

(ii) When $t=2$ and $2 \leq w \leq L-2$, we have that

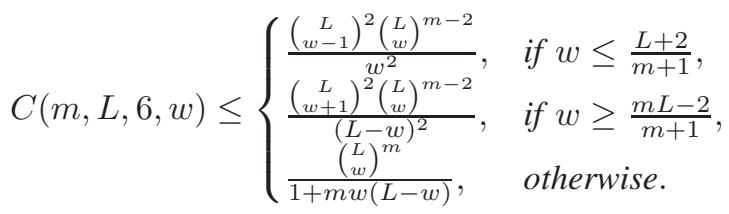

Proof. When $t=1$, we have three $m$-tuples in the set $\mathcal{P}(m, L ; w, 1)$. We list the $m$-tuples in $\mathcal{P}(m, L ; w, 1)$ below with their corresponding orbit sizes and entries in the matrix $M^{*}$.

\begin{tabular}{|c|c|c|}
\hline $\boldsymbol{u}$ & $O_{\boldsymbol{u}}$ & $\boldsymbol{M}_{\boldsymbol{w}, \boldsymbol{u}}^{*}$ \\
\hline$[w, w, \ldots, w]$ & $\left.\begin{array}{c}\text { ( } \\
w\end{array}\right)^{m}$ & 1 \\
{$[w+1, w, \ldots, w]$} & $m\left(\begin{array}{c}L \\
w+1\end{array}\right)\left(\begin{array}{c}L \\
w\end{array}\right)^{m-1}$ & $m(L-w)$ \\
{$[w, w, \ldots, w-1]$} & $m\left(\begin{array}{c}L \\
w-1\end{array}\right)\left(\begin{array}{c}L \\
w\end{array}\right)^{m-1}$ & $m w$ \\
\hline
\end{tabular}

Hence, (6) yields the value

$$
\min \left\{\frac{\left(\begin{array}{c}
L \\
w+1
\end{array}\right)\left(\begin{array}{c}
L \\
w
\end{array}\right)^{m-1}}{L-w},\left(\begin{array}{c}
L \\
w
\end{array}\right)^{m}, \frac{\left(\begin{array}{c}
L \\
w-1
\end{array}\right)\left(\begin{array}{c}
L \\
w
\end{array}\right)^{m-1}}{w}\right\} .
$$

Algebraic manipulations then yield (i).

For $t=2$, we proceed as before. The eight $m$-tuples in $\mathcal{P}(m, L ; w, 2)$ below with their corresponding orbit sizes and entries in the matrix $M^{*}$.

\begin{tabular}{|c|c|c|}
\hline $\boldsymbol{u}$ & $\left|O_{\boldsymbol{u}}\right|$ & $\boldsymbol{M}_{\boldsymbol{w}, \boldsymbol{u}}^{*}$ \\
\hline$[w, w, \ldots, w]$ & $\left(\begin{array}{c}L \\
w\end{array}\right)^{m}$ & $1+m L(L-w)$ \\
{$[w+1, w, \ldots, w]$} & $m\left(\begin{array}{c}L \\
w+1\end{array}\right)\left(\begin{array}{c}L \\
w\end{array}\right)^{m-1}$ & $m(L-w)$ \\
{$[w, w, \ldots, w-1]$} & $m\left(\begin{array}{c}L \\
w-1\end{array}\right)\left(\begin{array}{c}L \\
w\end{array}\right)^{m-1}$ & $m w$ \\
{$[w+2, w, \ldots, w]$} & $m\left(\begin{array}{c}L \\
w+1\end{array}\right)\left(\begin{array}{c}L \\
w\end{array}\right)$ & $m(L-w)$ \\
{$[w+1, w+1, \ldots, w]$} & $m\left(\begin{array}{c}L \\
w+1\end{array}\right)^{2}\left(\begin{array}{c}L \\
w\end{array}\right)^{m-2}$ & $\left(\begin{array}{c}m \\
2\end{array}\right)(L-w)^{2}$ \\
{$[w+1, w, \ldots, w-1]$} & $m(m-1)\left(\begin{array}{c}L \\
w+1\end{array}\right)\left(\begin{array}{c}L \\
w-1\end{array}\right)\left(\begin{array}{c}L \\
w\end{array}\right)$ & $m(m-1) w(L-w)$ \\
{$[w, \ldots, w-1, w-1]$} & $m\left(\begin{array}{c}L \\
w-1\end{array}\right)^{2}\left(\begin{array}{c}L \\
w\end{array}\right)^{m-2}$ & $\left(\begin{array}{c}m \\
2\end{array}\right) w^{2}$ \\
{$[w, w, \ldots, w-2]$} & $m\left(\begin{array}{c}L \\
w+1\end{array}\right)\left(\begin{array}{c}L \\
w\end{array}\right)$ & $m(L-1$ \\
\hline
\end{tabular}

As before, (6) and algebraic manipulations yield (ii).

\section{B. Subblock Energy Constrained Codes}

Consider the space $\mathcal{S}=\mathcal{S}(m, L, w)$ and set $t=\lfloor(d-1) / 2\rfloor$. Let $\mathcal{T}$ be the resulting space and again, our task is to determine the number of orbits that are contained in $\mathcal{T}$. To this end, we set

$$
\mathcal{P}_{\text {row }}(m, L ; w) \triangleq\left\{\boldsymbol{v} \in \mathcal{P}(m, L): L \geq v_{1} \geq \cdots \geq v_{m} \geq w\right\} .
$$


Hence, $\mathcal{S}(m, L, w)=\bigcup_{\boldsymbol{v} \in \mathcal{P}_{\text {row }}(m, L ; w)} O_{\boldsymbol{v}}$. As before, we define $\mathcal{P}(m, L ; \boldsymbol{v}, t)=\left\{\boldsymbol{u} \in \mathcal{P}(m, L): \sum_{i=1}^{m}\left|u_{i}-v_{i}\right| \leq t\right\}$. Hence, the orbits that partition $\mathcal{T}$ have indices in the set

$$
\mathcal{P}_{\text {col }}(m, L ; w, t) \triangleq \bigcup_{\boldsymbol{v} \in \mathcal{P}_{\text {row }}(m, L ; w)} \mathcal{P}(m, L ; \boldsymbol{v}, t) .
$$

The next proposition states that $N(t)$ (defined in (4)) is an upper bound for $\mathcal{P}(m, L ; \boldsymbol{v}, t)$. This in turn provides an upper bound for $\mathcal{P}_{\text {col }}(m, L ; w, t)$, the number of variables in the reduced program. The proof of Proposition 7 is deferred to Appendix $\mathrm{A}$.

Proposition 7. For all $m, L, \boldsymbol{v}, t$, we have $|\mathcal{P}(m, L ; \boldsymbol{v}, t)| \leq N(t)$. Therefore, $\mathcal{P}_{\text {col }}(m, L ; w, t) \leq L^{m} N(t)$.

Applying Theorem 3, we reduce (1) to the following optimization program.

$$
\min \left\{\sum_{\boldsymbol{u} \in \mathcal{P}_{\mathrm{col}}(m, L ; w, t)}\left|O_{\boldsymbol{u}}\right| Y_{\boldsymbol{u}}^{*}: \boldsymbol{M}^{*} \boldsymbol{Y}^{*} \geq \mathbf{1}, Y_{\boldsymbol{u}} \geq 0\right\},
$$

$\boldsymbol{M}^{*}$ is the matrix indexed by $\mathcal{P}_{\text {row }}(m, L ; w) \times \mathcal{P}_{\text {col }}(m, L ; w, t)$ whose entries are given by

$$
\boldsymbol{M}_{\boldsymbol{u}, \boldsymbol{v}}^{*} \triangleq\left|B_{O_{\boldsymbol{v}}}(\boldsymbol{x}, t)\right| \text { for some } \boldsymbol{x} \in O_{\boldsymbol{u}}
$$

Example 3. Consider $m=4, L=3, w=2$ and $t=1$. Then

$$
\begin{aligned}
\mathcal{P}_{\text {row }}(4,3 ; 2) & =\{[3,3,3,3],[3,3,3,2],[3,3,2,2],[3,2,2,2],[2,2,2,2]\} \\
\mathcal{P}_{\text {col }}(4,3 ; 2,1) & =\mathcal{P}_{\text {row }}(4,3 ; 2) \cup\{[3,3,3,1],[3,3,2,1],[3,2,2,1],[2,2,2,1]\} .
\end{aligned}
$$

Then $M^{*}$ is given by

$$
\boldsymbol{M}^{*}=\left(\begin{array}{rrrrrrrrr}
1 & 12 & 0 & 0 & 0 & 0 & 0 & 0 & 0 \\
1 & 1 & 9 & 0 & 0 & 2 & 0 & 0 & 0 \\
0 & 2 & 1 & 6 & 0 & 0 & 4 & 0 & 0 \\
0 & 0 & 3 & 1 & 3 & 0 & 0 & 6 & 0 \\
0 & 0 & 0 & 4 & 1 & 0 & 0 & 0 & 8
\end{array}\right)
$$

and the objective function is given by

$$
Y_{3333}+12 Y_{3332}+54 Y_{3322}+108 Y_{3222}+81 Y_{2222}+12 Y_{3331}+108 Y_{3321}+324 Y_{3221}+324 Y_{2221} .
$$

Since the number of orbits contained in $\mathcal{S}(m, L ; w)$ is at most $L^{m}$, the optimization problem (7) for SECCs has at most $L^{m} N(t)$ variables and at most $L^{m}$ constraints. Therefore, we have the following proposition.

Proposition 8. For all $m, L, w, t$, the exact solution to 11) for SECCs can be computed in time polynomial in $L^{m}$ and $N(t)$.

Even though we reduce the number of variables from $\Omega\left(2^{m L}\right)$ to $O\left(L^{m} N(t)\right)$, the number of variables remains exponential in $m$. Nevertheless, when $t=1$, we are able to provide closed-form solutions for the optimization problem (7).

To solve the linear program, we introduce the notion of optimality certificates.

Definition 9. A pair $\left(\tilde{\boldsymbol{Y}}^{*}, \widetilde{\boldsymbol{X}}^{*}\right)$ is an optimality certificate for (7) if the following holds:

(i) $\widetilde{\boldsymbol{Y}}^{*}$ is feasible solution for the primal problem. In other words, $\boldsymbol{M}^{*} \tilde{\boldsymbol{Y}}^{*} \geq \mathbf{1}$ and $\tilde{Y}_{\boldsymbol{u}}^{*} \geq 0$ for all $\boldsymbol{u} \in \mathcal{P}_{\text {col }}(m, L ; w, t)$.

(ii) $\widetilde{\boldsymbol{X}}^{*}$ is feasible solution for the dual problem. In other words, $\widetilde{\boldsymbol{X}}^{*} \boldsymbol{M}^{*} \leq \boldsymbol{O}$ and $\widetilde{X}_{\boldsymbol{u}}^{*} \geq 0$ for $\boldsymbol{u} \in \mathcal{P}_{\text {row }}(m, L ; w)$. Here, $\boldsymbol{O}=\left(\left|O_{\boldsymbol{u}}\right|\right)_{\boldsymbol{u} \in \mathcal{P}_{\mathrm{col}}(m, L ; w, t)}$.

(iii) $\sum_{\boldsymbol{u} \in \mathcal{P}_{\text {col }}(m, L ; w, t)}\left|O_{\boldsymbol{u}}\right| \tilde{Y}_{\boldsymbol{u}}^{*}=\sum_{\boldsymbol{u} \in \mathcal{P}_{\text {row }}(m, L ; w)} \tilde{X}_{\boldsymbol{u}}^{*}$.

Given an optimality certificate, it is then straightforward to obtain the optimal value.

Proposition 10 (see Chvatal [14]). If $\left(\widetilde{\boldsymbol{Y}}^{*}, \widetilde{\boldsymbol{X}}^{*}\right)$ is an optimality certificate for (7), then the optimal value for (7) is given by $\sum_{\boldsymbol{u} \in \mathcal{P}(m, L ; w, t)}\left|O_{\boldsymbol{u}}\right| \widetilde{Y}_{\boldsymbol{u}}^{*}$

Example 4 (Example 3 continued). Consider $m=4, L=3, w=2$ and $t=1$. Then consider the pair $\left(\widetilde{\boldsymbol{Y}}^{*}, \widetilde{\boldsymbol{X}}^{*}\right)$, where

$$
\begin{aligned}
& \widetilde{\boldsymbol{Y}}^{*}=(0,1 / 12,1 / 4,1 / 4,0,0,0,0,0), \text { and } \\
& \widetilde{\boldsymbol{X}}^{*}=(1,0,0,18,45 / 2) .
\end{aligned}
$$

We verify that

$$
\boldsymbol{M}^{*} \widetilde{\boldsymbol{Y}}^{*} \geq \mathbf{1} \text { and } \widetilde{\boldsymbol{X}}^{*} \boldsymbol{M}^{*} \leq(1,12,54,108,81,12,108,324,324)
$$


Also, we check that

$$
\begin{aligned}
\sum_{\boldsymbol{u} \in \mathcal{P}_{\mathrm{col}}(m, L ; w, t)}\left|O_{\boldsymbol{u}}\right| \tilde{Y}_{\boldsymbol{u}}^{*} & =\frac{12}{12}+\frac{54}{4}+\frac{108}{4}=41.5 \\
\sum_{\boldsymbol{u} \in \mathcal{P}_{\text {row }}(m, L ; w)} \tilde{X}_{\boldsymbol{u}}^{*} & =1+18+\frac{45}{2}=41.5
\end{aligned}
$$

Hence, $\left(\widetilde{\boldsymbol{Y}}^{*}, \widetilde{\boldsymbol{X}}^{*}\right)$ satisfies all properties in Definition 9 Therefore, it follows from Proposition 10 that the solution to (10 for SECCs is 41.5 .

Therefore, to determine (7), it suffices to provide optimality certificates for the problem. We provide these certificates and the detailed verification in Appendix B] Here, we state the exact solutions to (7).

Proposition 11. Fix $t=1$ and $w=L-1$. For all $L$ and $L \geq m / 2$, the exact solution to (7)is as follow.

(i) When $m \equiv 0(\bmod 4)$, the solution is

$$
1+\sum_{i=0}^{\lfloor m / 4\rfloor-1} \frac{1}{4 i+4}\left(\left(\begin{array}{c}
m \\
4 i+2
\end{array}\right) L^{4 i+2}+\left(\begin{array}{c}
m \\
4 i+3
\end{array}\right) L^{4 i+3}\right)
$$

(ii) when $m \equiv 1(\bmod 4)$, the solution is

$$
1+\sum_{i=0}^{\lfloor m / 4\rfloor-1} \frac{1}{4 i+5}\left(\left(\begin{array}{c}
m \\
4 i+3
\end{array}\right) L^{4 i+3}+\left(\begin{array}{c}
m \\
4 i+4
\end{array}\right) L^{4 i+4}\right)
$$

(iii) when $m \equiv 2(\bmod 4)$, the solution is

$$
\sum_{i=0}^{\lfloor m / 4\rfloor} \frac{1}{4 i+2}\left(\left(\begin{array}{c}
m \\
4 i
\end{array}\right) L^{4 i}+\left(\begin{array}{c}
m \\
4 i+1
\end{array}\right) L^{4 i+1}\right)
$$

(iv) when $m \equiv 3(\bmod 4)$, the solution is

$$
\sum_{i=0}^{\lfloor m / 4\rfloor} \frac{1}{4 i+3}\left(\left(\begin{array}{c}
m \\
4 i+1
\end{array}\right) L^{4 i+1}+\left(\begin{array}{c}
m \\
4 i+2
\end{array}\right) L^{4 i+2}\right) .
$$

Proposition 12. Fix $t=1$ and $m=1$. For all $L$ and $w \geq L / 2$, the exact solution to (7) is as follow.

(i) When $L-w \equiv 0(\bmod 4)$, the solution is

$$
1+\sum_{i=0}^{\lfloor(L-w) / 4\rfloor-1} \frac{1}{4 i+4}\left(\left(\begin{array}{c}
L \\
4 i+2
\end{array}\right)+\left(\begin{array}{c}
L \\
4 i+3
\end{array}\right)\right)
$$

(ii) when $L-w \equiv 1(\bmod 4)$, the solution is

$$
1+\sum_{i=0}^{\lfloor(L-w) / 4\rfloor} \frac{1}{4 i+5}\left(\left(\begin{array}{c}
L \\
4 i+3
\end{array}\right)+\left(\begin{array}{c}
L \\
4 i+4
\end{array}\right)\right)
$$

(iii) when $L-w \equiv 2(\bmod 4)$, the solution is

$$
\sum_{i=0}^{\lfloor(L-w) / 4\rfloor} \frac{1}{4 i+2}\left(\left(\begin{array}{c}
L \\
4 i
\end{array}\right)+\left(\begin{array}{c}
L \\
4 i+1
\end{array}\right)\right)
$$

(iv) when $L-w \equiv 3(\bmod 4)$, the solution is

$$
\sum_{i=0}^{\lfloor(L-w) / 4\rfloor} \frac{1}{4 i+3}\left(\left(\begin{array}{c}
L \\
4 i+1
\end{array}\right)+\left(\begin{array}{c}
L \\
4 i+2
\end{array}\right)\right) .
$$




\section{IMPROVED BOUNDS ON ASYMPTOTIC RATES}

In this section, we provide improved upper bounds on the asymptotic rates for CSCCs and SECCs for a range of relative distance values. These results are obtained by judiciously choosing appropriate constrained spaces for estimating asymptotic ball sizes, and by applying the generalized sphere-packing bound.

\section{A. Constant Subblock-Composition Codes}

We present bounds for the CSCC rate in the asymptotic setting where the number of subblocks $m$ tends to infinity, minimum distance $d$ scales linearly with $m$, but $L$ and $w$ are fixed. Formally, for fixed $0<\delta<1$, the asymptotic rate for CSCCs with fixed subblock length $L$, subblock weight parameter $w$, number of subblocks in a codeword $m \rightarrow \infty$, and minimum distance $d$ scaling as $d=\lfloor m L \delta\rfloor$ is defined as

$$
\gamma(L, \delta, w / L) \triangleq \limsup _{m \rightarrow \infty} \frac{\log C(m, L,\lfloor m L \delta\rfloor, w)}{m L} .
$$

The asymptotic CSCC rate, $\gamma(L, \delta, w / L)$, was studied in [6] and it was shown that $\gamma(L, \delta, w / L)=0$ when $\delta \geq \delta^{*}(w / L)$, where $\delta^{*}(w / L)$ is defined as

$$
\delta^{*}(w / L) \triangleq 2\left(\frac{w}{L}\right)\left(1-\frac{w}{L}\right)
$$

Further, in [6] the following sphere-packing upper bound on $\gamma(L, \delta, w / L)$ was presented.

Theorem 13 (Tandon et al. [6]). For $0<\delta<\delta^{*}(w / L)$, we have

$$
\gamma(L, \delta, w / L) \leq \gamma_{S P}(L, \delta, w / L)
$$

where $\gamma_{S P}(L, \delta, w / L)$ is defined as

$$
\begin{aligned}
& \frac{1}{L} \log \left(\begin{array}{c}
L \\
w
\end{array}\right)-\left(\frac{1+\tilde{u}-\lceil\tilde{u}\rceil}{L}\right) \log \left(\begin{array}{c}
w \\
\lceil\tilde{u}\rceil
\end{array}\right) \\
& -\left(\frac{\lceil\tilde{u}\rceil \tilde{u}}{L}\right) \log \left(\begin{array}{c}
w \\
\lfloor\tilde{u}\rfloor
\end{array}\right)-\left(\frac{\lceil\tilde{u}\rceil-\tilde{u}}{L}\right) \log \left(\begin{array}{c}
L-w \\
\lfloor\tilde{u}\rfloor
\end{array}\right) \\
& -\left(\frac{1+\tilde{u}-\lceil\tilde{u}\rceil}{L}\right) \log \left(\begin{array}{c}
L-w \\
\lceil\tilde{u}\rceil
\end{array}\right)-\frac{1}{L} h(\lceil\tilde{u}\rceil-\tilde{u}),
\end{aligned}
$$

where $\tilde{u} \triangleq \delta L / 4$.

We will show that for certain parameters, the above result can be improved by applying the generalized sphere packing formulation in Theorem [2. The bound on the asymptotic CSCC rate in Theorem 13 was obtained by estimating the ball size in the space $\mathcal{C}(m, L, w)$, and therefore corresponds to the case where $\tilde{\mathcal{S}}=\mathcal{S}=\mathcal{C}(m, L, w)$. In Prop. 14 we present an upper bound on the optimal CSCC code-size, $C(m, L, d, w)$, by choosing the space $\tilde{\mathcal{S}}=\mathcal{C}(m, L, w+1)$.

Proposition 14. For $2 m<d \leq 6 m$ and $L \geq w+2$, with $t=\lfloor(d-1) / 2\rfloor$ and $\tilde{t}=\lfloor(t-m) / 2\rfloor$, we have

$$
C(m, L, d, w) \leq \frac{\left(\begin{array}{c}
L \\
w+1
\end{array}\right)^{m}}{\left(\begin{array}{c}
m \\
\tilde{t}
\end{array}\right)\left[\left(\begin{array}{c}
L-w \\
2
\end{array}\right)\left(\begin{array}{c}
w \\
1
\end{array}\right)\right]^{\tilde{t}}(L-w)^{m-\tilde{t}}} .
$$

Proof: We will apply Theorem 2 , where we choose $\tilde{\mathcal{S}}=\mathcal{C}(m, L, w+1)$. Thus $|\tilde{\mathcal{S}}|=\left(\begin{array}{c}L \\ w+1\end{array}\right)^{m}$, and using Theorem 2 it suffices to show that

$$
V_{\mathcal{S}, \tilde{\mathcal{S}}}^{\min }(t) \geq\left(\begin{array}{c}
m \\
\tilde{t}
\end{array}\right)\left[\left(\begin{array}{c}
L-w \\
2
\end{array}\right)\left(\begin{array}{c}
w \\
1
\end{array}\right)\right]^{\tilde{t}}(L-w)^{m-\tilde{t}},
$$

where the constrained CSCC space is $\mathcal{S}=\mathcal{C}(m, L, w)$. For $\boldsymbol{x} \in \mathcal{S}$, let $\Lambda_{\boldsymbol{x}}$ consist of all words $\boldsymbol{y} \in \tilde{\mathcal{S}}$ which satisfy the following two properties:

(i) $\tilde{t}$ subblocks of $\boldsymbol{y}$ differ from corresponding subblocks of $\boldsymbol{x}$ in exactly three bit positions.

(ii) Remaining $m-\tilde{t}$ subblocks of $\boldsymbol{y}$ differ from corresponding subblocks of $\boldsymbol{x}$ in exactly one bit position.

The size of $\Lambda_{\boldsymbol{x}}$ is given by

$$
\left|\Lambda_{\boldsymbol{x}}\right|=\left(\begin{array}{c}
m \\
\tilde{t}
\end{array}\right)\left[\left(\begin{array}{c}
L-w \\
2
\end{array}\right)\left(\begin{array}{c}
w \\
1
\end{array}\right)\right]^{\tilde{t}}(L-w)^{m-\tilde{t}} .
$$

For any $\boldsymbol{y} \in \Lambda_{\boldsymbol{x}}$, we observe that $\tau(\boldsymbol{x}, \boldsymbol{y})=3 \tilde{t}+(m-\tilde{t}) \leq t$, and thus $\Lambda_{\boldsymbol{x}} \subseteq \mathcal{B}_{\tilde{\mathcal{S}}}(\boldsymbol{x}, t)$. Finally, the inequality in (12) follows because $\mathcal{B}_{\tilde{\mathcal{S}}}(\boldsymbol{x}, t) \geq\left|\Lambda_{\boldsymbol{x}}\right|$ for all $\boldsymbol{x} \in \mathcal{S}$.

The following theorem applies Prop. 14 to provide an upper bound on the asymptotic rate for CSCCs. 


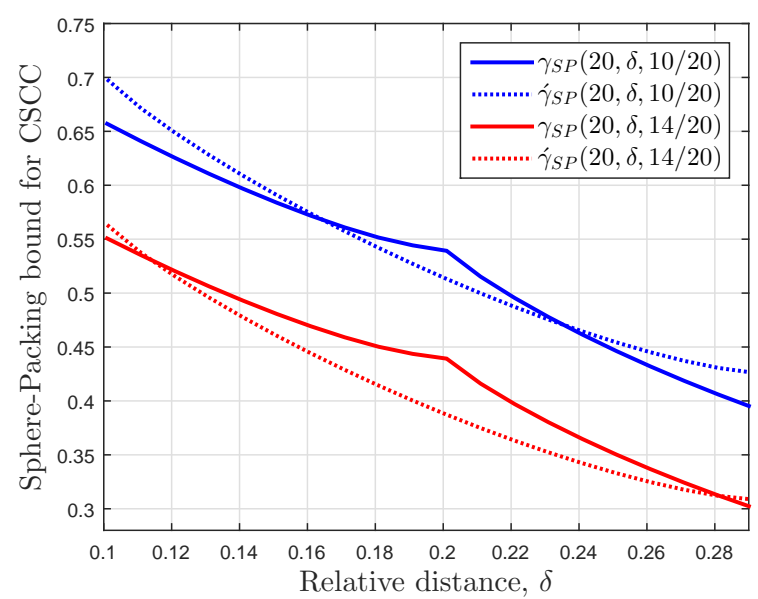

Fig. 1: Comparison of sphere-packing upper bounds for the CSCC asymptotic rate $\gamma(L, \delta, w / L)$ for $L=20$.

Theorem 15. For $2 / L<\delta<6 / L \leq \delta^{*}(w / L)$, we have

$$
\gamma(L, \delta, w / L) \leq \dot{\gamma}_{S P}(L, \delta, w / L)
$$

where $\dot{\gamma}_{S P}(L, \delta, w / L)$ is defined as

$$
\begin{array}{r}
\frac{1}{L} \log \left(\begin{array}{c}
L \\
w+1
\end{array}\right)-\left(\frac{\delta}{4}-\frac{1}{2 L}\right) \log \left[\left(\begin{array}{c}
L-w \\
2
\end{array}\right)\left(\begin{array}{c}
w \\
1
\end{array}\right)\right] \\
-\frac{1}{L} h\left(\frac{L \delta}{4}-\frac{1}{2}\right)-\left(\frac{3}{2 L}-\frac{\delta}{4}\right) \log (L-w) .
\end{array}
$$

Proof: We will combine (8) and (11) to prove the theorem. Towards this, note that when $d$ scales as $d=\lfloor m L \delta\rfloor$, and $\tilde{t}=\lfloor(t-m) / 2\rfloor$ with $t=\lfloor(d-1) / 2\rfloor$, then we have

$$
\begin{aligned}
\limsup _{m \rightarrow \infty} \frac{1}{m L} \log \left[\left(\begin{array}{c}
m \\
\tilde{t}
\end{array}\right)\right] & =\frac{1}{L} h\left(\frac{L \delta}{4}-\frac{1}{2}\right), \\
\limsup _{m \rightarrow \infty} \frac{\tilde{t}}{m L} & =\left(\frac{\delta}{4}-\frac{1}{2 L}\right), \\
\limsup _{m \rightarrow \infty} \frac{m-\tilde{t}}{m L} & =\left(\frac{3}{2 L}-\frac{\delta}{4}\right) .
\end{aligned}
$$

The proof is now complete by combining (15), 116, (17), with (8) and (11).

Proposition 16. For $L / 2 \leq w<L-1$ and $\delta=4 / L$, we have

$$
\dot{\gamma}_{S P}(L, \delta, w / L)<\gamma_{S P}(L, \delta, w / L)
$$

Proof: When $\delta=4 / L$, using (10) we get

$$
\gamma_{S P}(L, 4 / L, w / L)=\frac{1}{L} \log \left(\begin{array}{c}
L \\
w
\end{array}\right)-\frac{1}{L} \log ((L-w) w) .
$$

On the other hand, using (14) we observe that $\dot{\gamma}_{S P}(L, 4 / L, w / L)$ is equal to

$$
\begin{aligned}
& \frac{1}{L} \log \left(\begin{array}{c}
L \\
w+1
\end{array}\right)-\frac{1}{2 L} \log \left(2(L-w)^{2}(L-w-1) w\right) \\
= & \frac{1}{L} \log \left(\begin{array}{c}
L \\
w
\end{array}\right)-\frac{1}{2 L} \log \left(2(w+1)^{2}(L-w-1) w\right) .
\end{aligned}
$$

The proposition is now proved by comparing (18) and (19), and observing that $2 w(L-w-1) \geq(L-w)^{2}$ when $L / 2 \leq w<L-1$.

Remark: As $\dot{\gamma}_{S P}(L, \delta, w)$ and $\gamma_{S P}(L, \delta, w)$ are both continuous functions of $\delta$, we observe that Prop. 16implies that for a certain interval around $\delta=4 / L$, the upper bound on the CSCC asymptotic rate given by $\hat{\gamma}_{S P}(L, \delta, w)$ is an improved upper bound on the CSCC rate compared to $\gamma_{S P}(L, \delta, w)$. This is depicted in Fig. 1 for the case where $L=20$ and $w \in\{10,14\}$. Fig. 1 shows that $\dot{\gamma}_{S P}(L, \delta, w)<\gamma_{S P}(L, \delta, w)$ for a range of $\delta$ values around $\delta=4 / L=0.2$. 


\section{B. Subblock Energy-Constrained Codes}

We provide an upper bound on the asymptotic SECC rate when the number of subblocks $m$ tends to infinity, minimum distance $d$ scales linearly with $m$, and parameters $L, w$ are fixed. Formally, for fixed $0<\delta<1$, the asymptotic rate for SECCs is defined as

$$
\sigma(L, \delta, w / L) \triangleq \limsup _{m \rightarrow \infty} \frac{\log S(m, L,\lfloor m L \delta\rfloor, w)}{m L} .
$$

Further, we introduce the notation $\left(\begin{array}{c}L \\ \geq w\end{array}\right)$ which we define as

$$
\left(\begin{array}{c}
L \\
\geq w
\end{array}\right) \triangleq \sum_{j=w}^{L}\left(\begin{array}{c}
L \\
j
\end{array}\right)
$$

First, we apply Theorem 2 to present an upper bound on the optimal code size for SECCs.

Proposition 17. For $d \leq 2 m+1,0 \leq m_{0} \leq m$, and $t=\lfloor(d-1) / 2\rfloor$, we have

$$
S(m, L, d, w) \leq \frac{\left(\begin{array}{c}
L \\
\geq w-1
\end{array}\right)^{m_{0}}\left(\begin{array}{c}
L \\
\geq w
\end{array}\right)^{m-m_{0}}}{\sum_{\substack{t_{1}, t_{2} \\
t_{1}+t_{2} \leq t}}\left(\begin{array}{c}
m_{0} \\
t_{1}
\end{array}\right)\left(\begin{array}{c}
m-m_{0} \\
t_{2}
\end{array}\right) L^{t_{1}}(L-w)^{t_{2}}} .
$$

Proof: We will apply Theorem 2 , and choose $\tilde{\mathcal{S}} \subset\{0,1\}^{m L}$ to be the space where the first $m_{0}$ subblocks have weight at least $w-1$, and the remaining $m-m_{0}$ subblocks have weight at least $w$, with fixed subblock length $L$. Thus $|\tilde{\mathcal{S}}|=\left(\begin{array}{c}L \\ \geq w-1\end{array}\right)^{m_{0}}\left(\begin{array}{c}L \\ \geq w\end{array}\right)^{m-m_{0}}$, and using Theorem 2 , it suffices to show that

$$
V_{\mathcal{S}, \tilde{\mathcal{S}}}^{\min }(t) \geq \sum_{\substack{t_{1}, t_{2} \\
t_{1}+t_{2} \leq t}}\left(\begin{array}{c}
m_{0} \\
t_{1}
\end{array}\right)\left(\begin{array}{c}
m-m_{0} \\
t_{2}
\end{array}\right) L^{t_{1}}(L-w)^{t_{2}} .
$$

For $\boldsymbol{x} \in \mathcal{S}(m, L, w)$, let $\boldsymbol{x}_{[i]}$ denote the $i$ th subblock of $\boldsymbol{x}$, and hence $\boldsymbol{x}=\left(\boldsymbol{x}_{[1]} \boldsymbol{x}_{[2]} \ldots \boldsymbol{x}_{[m]}\right)$. Let $\Lambda_{\boldsymbol{x}}$ be defined as

$$
\Lambda_{\boldsymbol{x}} \triangleq\left\{\boldsymbol{y} \in \tilde{\mathcal{S}}: \tau(\boldsymbol{x}, \boldsymbol{y}) \leq t, \tau\left(\boldsymbol{x}_{[i]}, \boldsymbol{y}_{[i]}\right) \leq 1, i \in\{1, \ldots, m\}\right\}
$$

Let $\boldsymbol{y} \in \tilde{\mathcal{S}}$ be such that $t_{1}$ (resp. $t_{2}$ ) subblocks out of the first $m_{0}$ (resp. last $m-m_{0}$ ) subblocks of $\boldsymbol{y}$ differ in exactly one bit from corresponding subblocks of $\boldsymbol{x}$, with $t_{1}+t_{2} \leq t$. Then $\boldsymbol{y} \in \Lambda_{\boldsymbol{x}}$, and

$$
\left|\Lambda_{\boldsymbol{x}}\right| \geq \sum_{\substack{t_{1}, t_{2} \\
t_{1}+t_{2} \leq t}}\left(\begin{array}{c}
m_{0} \\
t_{1}
\end{array}\right)\left(\begin{array}{c}
m-m_{0} \\
t_{2}
\end{array}\right) L^{t_{1}}(L-w)^{t_{2}} .
$$

Note that the inequality above holds for every $\boldsymbol{x} \in \mathcal{S}(m, L, w)$. Finally, the inequality in 22 follows because $\Lambda_{\boldsymbol{x}} \subseteq \mathcal{B}_{\tilde{\mathcal{S}}}(\boldsymbol{x}, t)$ for every $\boldsymbol{x} \in \mathcal{S}(m, L, w)$.

The following theorem gives an upper bound on the SECC rate $\sigma(L, \delta, w / L)$.

Theorem 18. For $0<\delta<2 / L$, we have

$$
\sigma(L, \delta, w / L) \leq R_{1}-\hat{\alpha} \nu
$$

where

$$
\begin{aligned}
R_{1} & \triangleq \frac{\log \left(\begin{array}{c}
L \\
\geq w
\end{array}\right)}{L}-\frac{h(\delta L / 2)}{L}-\frac{\delta}{2} \log (L-w) \\
h(x) & \triangleq-x \log (x)-(1-x) \log (1-x) \\
\nu & \triangleq \frac{\delta}{2} \log \left(\frac{L}{L-w}\right)-\frac{1}{L} \log \left[\frac{\left(\begin{array}{c}
L \\
\geq w-1
\end{array}\right)}{\left(\begin{array}{c}
L \\
\geq w
\end{array}\right]}\right. \\
\hat{\alpha} & \triangleq\left\{\begin{array}{l}
0, \text { if } \nu \leq 0 \\
1, \text { if } \nu>0
\end{array}\right.
\end{aligned}
$$

Proof: For $0 \leq m_{0} \leq m$, let $\alpha=m_{0} / m$ with $t_{1}=\lfloor t \alpha\rfloor$ and $t_{2}=\lfloor t(1-\alpha)\rfloor$. Then $t_{1}+t_{2} \leq t$, and it follows from (21) that

$$
S(m, L, d, w) \leq \frac{\left(\begin{array}{c}
L \\
\geq w-1
\end{array}\right)^{m_{0}}\left(\begin{array}{c}
L \\
\geq w
\end{array}\right)^{m-m_{0}}}{\left(\begin{array}{c}
m_{0} \\
\lfloor\alpha\rfloor
\end{array}\right)\left(\begin{array}{c}
m-m_{0} \\
\lfloor t(1-\alpha)\rfloor
\end{array}\right) L^{\lfloor t \alpha\rfloor}(L-w)^{\lfloor t(1-\alpha)\rfloor}},
$$




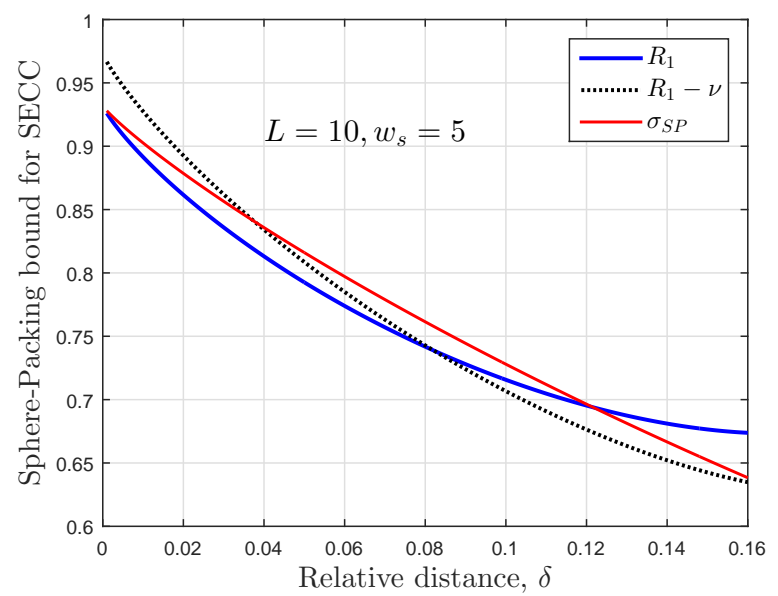

Fig. 2: Comparison of sphere-packing upper bounds for the SECC asymptotic rate $\sigma(L, \delta, w / L)$.

Combining 20) and 27, we get

$$
\begin{aligned}
\sigma(L, \delta, w / L) & \leq \frac{\alpha}{L} \log \left(\begin{array}{c}
L \\
\geq w-1
\end{array}\right)+\frac{1-\alpha}{L} \log \left(\begin{array}{c}
L \\
\geq w
\end{array}\right) \\
& -\frac{\alpha}{L} h\left(\frac{\delta L}{2}\right)-\frac{(1-\alpha)}{L} h\left(\frac{\delta L}{2}\right) \\
& -\frac{\alpha \delta}{2} \log (L)-\frac{(1-\alpha) \delta}{2} \log (L-w) .
\end{aligned}
$$

By combining the coefficients of $\alpha$, the above inequality can be expressed as

$$
\sigma(L, \delta, w / L) \leq R_{1}-\alpha \nu,
$$

where $R_{1}$ and $\nu$ are given by (24) and (25), respectively. The above bound on SECC rate holds for all $\alpha \in[0,1]$, and hence the right side in (28) is minimized by choosing $\alpha=\hat{\alpha}$, with $\hat{\alpha}$ given by (26).

We observe that the upper bound on $\sigma(L, \delta, w / L)$, given by Theorem 18 can equivalently be expressed as

$$
\sigma(L, \delta, w / L) \leq \min \left\{R_{1}, R_{1}-\nu\right\},
$$

where $R_{1}$ corresponds to the sphere packing bound on the asymptotic rate $\sigma(L, \delta, w / L)$ when space $\tilde{\mathcal{S}}$ is chosen to be $\tilde{\mathcal{S}}=$ $\mathcal{S}(m, L, w)$, while $R_{1}-\nu$ corresponds to the sphere packing bound when space $\tilde{\mathcal{S}}$ is chosen to be $\tilde{\mathcal{S}}=\mathcal{S}(m, L, w-1)$.

Corollary 19. $R_{1}-\nu$, the upper bound on SECC rate obtained by choosing $\tilde{\mathcal{S}}=\mathcal{S}(m, L, w-1)$, is less than $R_{1}$, the upper bound on SECC rate obtained by choosing $\tilde{\mathcal{S}}=\mathcal{S}(m, L, w)$, for the following range of $\delta$ values

$$
\frac{2}{L \log [L /(L-w)]} \log \left[\frac{\left(\begin{array}{c}
L \\
\geq w-1
\end{array}\right)}{\left(\begin{array}{c}
L \\
\geq w
\end{array}\right)}\right]<\delta<\frac{2}{L} .
$$

Proof: Follows from 25].

An alternate sphere-packing bound was presented in [6], where it was shown that $\sigma(L, \delta, w / L)$, the asymptotic rate for SECCs, is upper bounded by

$$
\sigma_{S P} \triangleq \frac{\log \left(\begin{array}{c}
L \\
\geq w
\end{array}\right)}{L}-\frac{h(\delta L / 4)}{L}-\frac{\delta}{4} \log ((L-w)(w+1)) .
$$

Fig. 2 compares different sphere-packing bounds for the SECC asymptotic rate $\sigma(L, \delta, w / L)$ as a function of $\delta$ with fixed $L=10$, and $w=5$. As shows in Cor. 19, it is observed in Fig. 2 that the upper bound given by $R_{1}-\nu$ is less than $R_{1}$ for $\delta>\frac{2}{L \log [L /(L-w)]} \log \left[\frac{\left(\begin{array}{c}L \\ \geq w-1\end{array}\right)}{\left(\begin{array}{l}L \\ \geq w\end{array}\right)}\right]=0.0821$. 


\section{CONCLUding REMARKS}

We study the generalized sphere-packing bound for two classes of subblock-constrained codes, namely, CSCCs and SECCs. Using automorphisms, we significantly reduce the number of variables in the associated linear programming problem.

For CSCCs, to determine the upper bound for $C(m, L, d, w)$, we show that the generalised sphere-packing bound can be obtained by finding the minimum of $N(t)$ values, where $t=\lfloor(d-1) / 2\rfloor$ and $N(t)$ is independent of $m, L$ and $w$. We then provide closedform solutions for the generalized sphere-packing bounds for single- and double-error correcting CSCCs in Corollary 6

In contrast, for SECCs, the generalised sphere-packing bound for $S(m, L, d, w)$ is obtained via a linear program involving at most $L^{m} N(t)$ variables. Nevertheless, in the special cases, we solved the linear program and closed-form solutions are provided in Propositions 11 and 12 .

Further, we extended the results in [6] to present improved upper bounds on the asymptotic rates for both CSCCs and SECCs for a range of relative distance values.

\section{APPENDIX A}

\section{UPPER BOUND ON THE NUMBER OF ORBITS}

In this appendix, we prove Propositions 4 and 7

Recall that $\mathcal{P}(m, L ; w, t) \triangleq\left\{\boldsymbol{u} \in \mathcal{P}(m, L): \sum_{i=1}^{m}\left|u_{i}-w\right| \leq t\right\}$ and our task is to provide an upper bound on the size of $\mathcal{P}(m, L ; w, t)$.

To this end, we consider the notion of partitions and partitions into parts of two kinds. Specifically, for a fixed value of $t$, we say that a tuple $\lambda=\left(\ell_{1}, \ell_{2}, \ldots, \ell_{s}\right)$ is a partition of $t$ if $\ell_{1} \geq \ell_{2} \geq \cdots \geq \ell_{s}>0$ and $\sum_{i=1}^{s} \ell_{i}=t$. A pair $\left(\lambda_{1}, \lambda_{2}\right)$ is a partition of $t$ into parts of two kinds if $\lambda_{i}$ is a partition of $t_{i}$ for $i \in\{1,2\}$ and $t_{1}+t_{2}=t$. We then set $\Lambda(t)$ to be the collection of all partitions of $t$ into parts of two kinds. The size of $\Lambda(t)$ is (see for example, sequence A000712 - https://oeis.org/A000712) as follow.

$$
|\Lambda(t)|=\sum_{i=0}^{t} p(i) p(t-i),
$$

where $p(i)$ is the partition number of $i$.

To give an upper bound on the size of $\mathcal{P}(m, L ; w, t)$, we define the map $\phi: \mathcal{P}(m, L ; w, t) \rightarrow \bigcup_{r=0}^{t} \Lambda(r)$. For $\boldsymbol{u} \in \mathcal{P}(m, L ; w, t)$, set $\left(\ell_{1}, \ell_{2}, \ldots, \ell_{m}\right)=\boldsymbol{u}-\boldsymbol{w}$ where $\boldsymbol{w}=[w, w, \ldots, w]$. Then we find $i$ and $j$ such that $\ell_{i-1}>0, \ell_{j+1}<0$ and $\ell_{i}=\ell_{i+1}=\ldots=$ $\ell_{j}=0$. Finally, we let $\lambda_{1}=\left(\ell_{1}, \ell_{2}, \ldots, \ell_{i-1}\right)$ and $\lambda_{2}=\left(-\ell_{m},-\ell_{m-1}, \ldots,-\ell_{j+1}\right)$, and set $\phi(\boldsymbol{u})=\left(\lambda_{1}, \lambda_{2}\right)$.

Lemma 20. Let $\phi: \mathcal{P}(m, L ; w, t) \rightarrow \bigcup_{r=0}^{t} \Lambda(r)$ be defined as above. Then $\phi$ is a well-defined injective map. Furthermore, if $m \geq t$, we have that $\phi$ is a bijection.

Proof. Let $\lambda_{1}$ and $\lambda_{2}$ be defined as above. Since $u_{1} \geq u_{2} \geq \cdots \geq u_{m}$, we have that $\lambda_{1}$ and $\lambda_{2}$ are partitions. Since $\sum_{i=1}^{m}\left|u_{i}-w\right|=$ $\sum_{i=1}^{m} \ell_{i}=r$, we have that $\left(\lambda_{1}, \lambda_{2}\right)$ is a partition of $r$ into parts of two kinds. As $r \leq t$, we have that $\left(\lambda_{1}, \lambda_{2}\right)$ belongs to $\bigcup_{r=0}^{t} \Lambda(r)$ and the map $\phi$ is therefore well-defined.

To demonstrate injectivity, suppose that $\left(\lambda_{1}, \lambda_{2}\right)$ is the image $\phi(\boldsymbol{u})$ and we find $\boldsymbol{u}$ from $\left(\lambda_{1}, \lambda_{2}\right)$. First, we pad $\lambda_{1}$ with $m-\left|\lambda_{1}\right|$ zeroes to obtain $\ell_{1}$. Next, we reverse $\lambda_{2}$ and pad the result $m-\left|\lambda_{2}\right|$ zeroes at the front to obtain $\ell_{2}$. It is then easy to verify that $\boldsymbol{u}=\boldsymbol{\ell}_{1}-\boldsymbol{\ell}_{2}+\boldsymbol{w}$.

Finally, we assume that $m \geq t$ and we consider $\left(\lambda_{1}, \lambda_{2}\right) \in \bigcup_{r=0}^{t} \Lambda(r)$. Since $\lambda_{1}$ and $\lambda_{2}$ are partitions of $t_{1}$ and $t_{2}$ with $t_{1}+t_{2} \leq t$, the sum of their lengths $\left|\lambda_{1}\right|+\left|\lambda_{2}\right|$ is at most $t$. Define $\ell_{1}$ and $\ell_{2}$ as in the preceding paragraph and we have that the support sets of $\ell_{1}$ and $\ell_{2}$ are disjoint. Therefore, the tuple $\boldsymbol{u}=\boldsymbol{\ell}_{1}-\boldsymbol{\ell}_{2}+\boldsymbol{w}$ has non-increasing entries and belong to $\mathcal{P}(m, L ; w, t)$. Since $\phi(\boldsymbol{u})=\left(\lambda_{1}, \lambda_{2}\right)$, the map $\phi$ is surjective and therefore, bijective.

(4) then follows from the fact that $\phi$ is injective and the formula 31 . To complete the proof of Proposition 4 we observe that $\phi$ is a bijection whenever $m \geq t$.

We next prove Propostion 7 Recall that $\mathcal{P}(m, L ; \boldsymbol{v}, t) \triangleq\left\{\boldsymbol{u} \in \mathcal{P}(m, L): \sum_{i=1}^{m}\left|u_{i}-v_{i}\right| \leq t\right\}$ and our task is to provide an upper bound on the size of $\mathcal{P}(m, L ; \boldsymbol{v}, t)$.

As before, we define the map $\phi_{\boldsymbol{v}}: \mathcal{P}(m, L ; w, t) \rightarrow \bigcup_{r=0}^{t} \Lambda(r)$. For $\boldsymbol{u} \in \mathcal{P}(m, L ; w, t)$, set $\left(\ell_{1}, \ell_{2}, \ldots, \ell_{m}\right)=\boldsymbol{u}-\boldsymbol{v}$. Then we find $i$ and $j$ such that $\ell_{i-1}>0, \ell_{j+1}<0$ and $\ell_{i}=\ell_{i+1}=\cdots=\ell_{j}=0$. Finally, we let $\lambda_{1}=\left(\ell_{1}, \ell_{2}, \ldots, \ell_{i-1}\right)$ and $\lambda_{2}=\left(-\ell_{m},-\ell_{m-1}, \ldots,-\ell_{j+1}\right)$, and set $\phi_{\boldsymbol{v}}(\boldsymbol{u})=\left(\lambda_{1}, \lambda_{2}\right)$. Following the proof for Lemma 20 , we obtain the following lemma.

Lemma 21. Let $\phi_{\boldsymbol{v}}: \mathcal{P}(m, L ; \boldsymbol{v}, t) \rightarrow \bigcup_{r=0}^{t} \Lambda(r)$ be defined as above. Then $\phi_{\boldsymbol{v}}$ is a well-defined injective map.

Therefore, Propostion 7 follows from the fact that $\phi_{\boldsymbol{v}}$ is injective and the formula 31. 


\begin{tabular}{|c|l|l|}
\hline$m$ & $\widetilde{\boldsymbol{Y}}^{*}$ & $\widetilde{\boldsymbol{X}}^{*}$ \\
\hline$m \equiv 0(\bmod 4)$ & $\widetilde{Y}_{L(m-1)}^{*}=\frac{1}{m L}$, & $\widetilde{X}_{L(m)}^{*}=1, \quad \widetilde{X}_{L(m-4 i-3)}^{*}=\frac{1}{4 i+3}\left(\begin{array}{c}m \\
4 i+2\end{array}\right) L^{4 i+2}$, \\
& $\widetilde{Y}_{L(m-4 i-2)}^{*}=\widetilde{Y}_{L(m-4 i-3)}^{*}=\frac{1}{4 i+4}$ for $0 \leq i<\left\lfloor\frac{m}{4}\right\rfloor$ & $\left.\widetilde{X}_{L(m-4 i-4)}^{*}=\frac{1}{4 i+4}\left(\begin{array}{c}m \\
4 i+3\end{array}\right) L^{4 i+3}-\frac{1}{4 i+3}\left(\begin{array}{c}m \\
4 i+2\end{array}\right) L^{4 i+2}\right)$ for $0 \leq i<\left\lfloor\frac{m}{4}\right\rfloor$ \\
\hline$m \equiv 1(\bmod 4)$ & $\widetilde{Y}_{L(m)}^{*}=1$, & $\widetilde{X}_{L(m)}^{*}=1, \quad \widetilde{X}_{L(m-4 i-4)}^{*}=\frac{1}{4 i+4}\left(\begin{array}{c}m \\
4 i+3\end{array}\right) L^{4 i+3}$, \\
& $\widetilde{Y}_{L(m-4 i-3)}^{*}=\widetilde{Y}_{L(m-4 i-4)}^{*}=\frac{1}{4 i+5}$ for $0 \leq i<\left\lfloor\frac{m}{4}\right\rfloor$ & $\left.\widetilde{X}_{L(m-4 i-5)}^{*}=\frac{1}{4 i+5}\left(\begin{array}{c}m \\
4 i+4\end{array}\right) L^{4 i+4}-\frac{1}{4 i+4}\left(\begin{array}{c}m \\
4 i+3\end{array}\right) L^{4 i+3}\right)$ for $0 \leq i<\left\lfloor\frac{m}{4}\right\rfloor$ \\
\hline$m \equiv 2(\bmod 4)$ & $\widetilde{Y}_{L(m-4 i)}^{*}=\widetilde{Y}_{L(m-4 i-1)}^{*}=\frac{1}{4 i+2}$ for $0 \leq i \leq\left\lfloor\frac{m}{4}\right\rfloor$ & $\widetilde{X}_{L(m-4 i-1)}^{*}=\frac{1}{4 i+1}\left(\begin{array}{c}m \\
4 i\end{array}\right) L^{4 i}$, \\
& & $\left.\widetilde{X}_{L(m-4 i-2)}^{*}=\frac{1}{4 i+2}\left(\begin{array}{c}m \\
4 i+1\end{array}\right) L^{4 i+1}-\frac{1}{4 i+1}\left(\begin{array}{c}m \\
4 i\end{array}\right) L^{4 i}\right)$ for $0 \leq i \leq\left\lfloor\frac{m}{4}\right\rfloor$ \\
\hline$m \equiv 3(\bmod 4)$ & $\widetilde{Y}_{L(m-4 i-1)}^{*}=\widetilde{Y}_{L(m-4 i-2)}^{*}=\frac{1}{4 i+3}$ for $0 \leq i \leq\left\lfloor\frac{m}{4}\right\rfloor$ & $\widetilde{X}_{L(m-4 i-2)}^{*}=\frac{1}{4 i+2}\left(\begin{array}{c}m \\
4 i+1\end{array}\right) L^{4 i+1}$, \\
& & $\left.\widetilde{X}_{L(m-4 i-3)}^{*}=\frac{1}{4 i+3}\left(\begin{array}{c}m \\
4 i+2\end{array}\right) L^{4 i+2}-\frac{1}{4 i+2}\left(\begin{array}{c}m \\
4 i+1\end{array}\right) L^{4 i+1}\right)$ for $0 \leq i \leq\left\lfloor\frac{m}{4}\right\rfloor$ \\
\hline
\end{tabular}

Here, we use $\boldsymbol{L}(i)$ to denote the $m$-tuple $[\underbrace{L, L, \ldots, L}_{i \text { times }}, \underbrace{L-1, L-1, \ldots, L-1}_{m-i \text { times }}]$.

TABLE I: List of Optimality Certificates for Proposition 11

\begin{tabular}{|c|c|c|}
\hline$L-w$ & $\tilde{\boldsymbol{Y}}^{*}$ & $\widetilde{\boldsymbol{X}}^{*}$ \\
\hline$L-w \equiv 0(\bmod 4)$ & $\begin{array}{l}\tilde{Y}_{[L]}^{*}=1, \\
\tilde{Y}_{[L-4 i-2]}^{*}=\tilde{Y}_{[L-4 i-3]}^{*}=\frac{1}{4 i+4} \text { for } 0 \leq i<\left|\frac{L-w}{4}\right|\end{array}$ & $\begin{array}{l}\widetilde{X}_{[L]}^{*}=1, \quad \widetilde{X}_{[L-4 i-3]}^{*}=\frac{1}{4 i+3}\left(\begin{array}{c}L \\
4 i+2\end{array}\right), \\
\widetilde{X}_{[L-4 i-4]}^{*}=\frac{1}{4 i+4}\left(\left(\begin{array}{c}L \\
4 i+3\end{array}\right)-\frac{1}{4 i+3}\left(\begin{array}{c}L \\
4 i+2\end{array}\right)\right) \text { for } 0 \leq i<\left\lfloor\frac{m}{4}\right\rfloor\end{array}$ \\
\hline$L-w \equiv 2(\bmod 4)$ & $\tilde{Y}_{[L-4 i]}^{*}=\widetilde{Y}_{[L-4 i-1]}^{*}=\frac{1}{4 i+2}$ for $0 \leq i \leq\left\lfloor\frac{L-w}{4}\right\rfloor$ & $\begin{aligned} \widetilde{X}_{[L-4 i-1]}^{*} & =\frac{1}{4 i+1}\left(\begin{array}{c}L \\
4 i\end{array}\right) \\
\widetilde{X}_{[L-4 i-2]}^{*} & =\frac{1}{4 i+2}\left(\left(\begin{array}{c}L \\
4 i+1\end{array}\right)-\frac{1}{4 i+1}\left(\begin{array}{c}L \\
4 i\end{array}\right)\right) \text { for } 0 \leq i \leq\left\lfloor\frac{L-w}{4} \mid\right.\end{aligned}$ \\
\hline$L-w \equiv 3(\bmod 4)$ & $\widetilde{Y}_{[L-4 i-1]}^{*}=\widetilde{Y}_{[L-4 i-2]}^{*}=\frac{1}{4 i+3}$ for $0 \leq i \leq\left\lfloor\frac{L-w}{4}\right\rfloor$ & $\begin{aligned} \widetilde{X}_{[L-4 i-2]}^{*} & =\frac{1}{4 i+2}\left(\begin{array}{c}L \\
4 i+1\end{array}\right) \\
\widetilde{X}_{[L-4 i-3]}^{*} & =\frac{1}{4 i+3}\left(\left(\begin{array}{c}L \\
4 i+2\end{array}\right)-\frac{1}{4 i+2}\left(\begin{array}{c}L \\
4 i+1\end{array}\right)\right) \text { for } 0 \leq i \leq \mid \frac{L-w}{4}\end{aligned}$ \\
\hline
\end{tabular}

TABLE II: List of Optimality Certificates for Proposition 12

\section{APPENDIX B \\ VERIFICATION OF OPTIMALITY CERTIFICATES}

We establish Propositions 11 and 12 by providing the optimality certificates for the linear program (7). In Tables 【 and II we provide the optimality certificates $\left(\widetilde{\boldsymbol{Y}}^{*}, \widetilde{\boldsymbol{X}}^{*}\right)$ for Propositions 11 and 12 respectively.

In this appendix, we also provide a detailed verification for the case $w=L-1, m \geq L / 2$, and $m \equiv 0$ (mod 4 ). We omit the detailed verification for the other cases as the verification process is similar.

For brevity, we adopt the following notations:

$$
\begin{aligned}
& \boldsymbol{L}(i) \triangleq[\underbrace{L, \ldots, L}_{i \text { times }}, \underbrace{L-1, \ldots, L-1}_{m-i \text { times }}], \\
& \boldsymbol{K}(i) \triangleq[\underbrace{L, \ldots, L}_{i \text { times }}, \underbrace{L-1, \ldots, L-1}_{m-i-1 \text { times }}, L-2] .
\end{aligned}
$$

We also write $\mathcal{P}_{\text {row }}(m, L ; w)$ and $\mathcal{P}_{\text {col }}(m, L ; w)$ as $\mathcal{P}_{\text {row }}$ and $\mathcal{P}_{\text {col }}$, respectively.

Observe that $\mathcal{P}_{\text {row }}=\{\boldsymbol{L}(i): 0 \leq i \leq m\}$ and $\mathcal{P}_{\text {col }}=\{\boldsymbol{L}(i): 0 \leq i \leq m\} \cup\{\boldsymbol{K}(i): 0 \leq i \leq m-1\}$. We now state explicitly the entries of $\boldsymbol{M}$. For $0 \leq i, j \leq m$, we have that

$$
\boldsymbol{M}_{\boldsymbol{L}(i), \boldsymbol{L}(j)}= \begin{cases}m-i, & \text { if } i=j-1, \\ 1, & \text { if } i=j, \\ L i, & \text { if } i=j+1, \\ 0, & \text { otherwise. }\end{cases}
$$

For $0 \leq i \leq m$ and $0 \leq j \leq m-1$, we have that

$$
\boldsymbol{M}_{\boldsymbol{L}(i), \boldsymbol{K}(j)}= \begin{cases}(L-1)(m-i), & \text { if } i=j, \\ 0, & \text { otherwise. }\end{cases}
$$


Next, we state explicitly the entries of $\boldsymbol{O}$.

$$
\begin{array}{lr}
\left|O_{\boldsymbol{L}(i)}\right|=\left(\begin{array}{c}
m \\
i
\end{array}\right) L^{m-i}, & \text { for } 0 \leq i \leq m, \\
\left|O_{\boldsymbol{K}(i)}\right|=m\left(\begin{array}{c}
m-1 \\
i
\end{array}\right) L^{m-i-1}\left(\begin{array}{c}
L \\
2
\end{array}\right), & \text { for } 0 \leq i \leq m-1 .
\end{array}
$$

We first verify property (i) for Definition 9 . In particular, we check that $\boldsymbol{M}^{*} \widetilde{\boldsymbol{Y}}^{*} \geq \mathbf{1}$, or equivalently, $\sum_{\boldsymbol{v} \in \mathcal{P}_{\text {col }}} \boldsymbol{M}_{\boldsymbol{L}(m-i), \boldsymbol{v}} \widetilde{Y}_{\boldsymbol{v}}^{*} \geq 1$ for all $0 \leq i \leq m$. Now, since all entries of $Y_{\boldsymbol{v}}$ are nonnegative, we have that

$$
\sum_{\boldsymbol{v} \in \mathcal{P}_{\mathrm{col}}} \boldsymbol{M}_{\boldsymbol{L}(m-i), \boldsymbol{v}} \tilde{Y}_{\boldsymbol{v}}^{*} \geq \sum_{j=i-1}^{i+1} \boldsymbol{M}_{\boldsymbol{L}(m-i), \boldsymbol{L}(m-j)} \tilde{Y}_{\boldsymbol{L}(m-j)}^{*}
$$

Then we have the following cases.

- When $i=0$, the quantity on the righthand side is bounded below by

$$
\boldsymbol{M}_{\boldsymbol{L}(m), \boldsymbol{L}(m-1)} \tilde{Y}_{\boldsymbol{L}(m-1)}^{*}=m L(1 / m L)=1 .
$$

- When $i \equiv 0(\bmod 4)$, the quantity on the righthand side is bounded below by

$$
\boldsymbol{M}_{\boldsymbol{L}(m-i), \boldsymbol{L}(m-i+1)} \tilde{Y}_{\boldsymbol{L}(m-i+1)}^{*}=i(1 / i)=1 .
$$

- When $i \equiv 1(\bmod 4)$, the quantity on the righthand side is bounded below by

$$
\boldsymbol{M}_{\boldsymbol{L}(m-i), \boldsymbol{L}(m-i-1)} \tilde{Y}_{\boldsymbol{L}(m-i-1)}^{*}=(m-i) L /(i+3) \geq 1 .
$$

- When $i \equiv 2(\bmod 4)$, the quantity on the righthand side is bounded below by

$$
\boldsymbol{M}_{\boldsymbol{L}(m-i), \boldsymbol{L}(m-i-1)} \tilde{Y}_{\boldsymbol{L}(m-i-1)}^{*}=(m-i) L /(i+2) \geq 1 .
$$

- When $i \equiv 3(\bmod 4)$, the quantity on the righthand side is bounded below by

$$
\begin{aligned}
\boldsymbol{M}_{\boldsymbol{L}(m-i), \boldsymbol{L}(m-i+1)} \tilde{Y}_{\boldsymbol{L}(m-i+1)}^{*}+\boldsymbol{M}_{\boldsymbol{L}(m-i), \boldsymbol{L}(m-i)} \tilde{Y}_{\boldsymbol{L}(m-i)}^{*} & \\
& =i /(i+1)+1 /(i+1)=1 .
\end{aligned}
$$

Next, we verify property (ii) for Definition 9 . In particular, we check that $\widetilde{\boldsymbol{X}}^{*} \boldsymbol{M}^{*} \leq \boldsymbol{O}$, or equivalently,

$$
\begin{array}{ll}
\sum_{\boldsymbol{v} \in \mathcal{P}_{\text {row }}} \tilde{X}_{\boldsymbol{v}}^{*} \boldsymbol{M}_{\boldsymbol{v}, \boldsymbol{L}(m-i)} \leq\left|O_{\boldsymbol{L}(m-i)}\right| & \text { for all } 0 \leq i \leq m, \\
\sum_{\boldsymbol{v} \in \mathcal{P}_{\text {row }}} \tilde{X}_{\boldsymbol{v}}^{*} \boldsymbol{M}_{\boldsymbol{v}, \boldsymbol{K}(m-i)} \leq\left|O_{\boldsymbol{K}(m-i)}\right| & \text { for all } 1 \leq i \leq m .
\end{array}
$$

Now, let us focus on the column indexed by $\boldsymbol{L}(m-i)$ for $0 \leq i \leq m$. Recall that $\left|O_{\boldsymbol{L}(m-i)}\right|=\left(\begin{array}{c}m \\ i\end{array}\right) L^{i}$. Since most entries of $\boldsymbol{M}$ are zero, we have that

$$
\sum_{\boldsymbol{v} \in \mathcal{P}_{\text {row }}} \tilde{X}_{\boldsymbol{v}}^{*} \boldsymbol{M}_{\boldsymbol{v}, \boldsymbol{L}(m-i)}=\sum_{j=i-1}^{i+1} \tilde{X}_{\boldsymbol{L}(m-j)}^{*} \boldsymbol{M}_{\boldsymbol{L}(m-j), \boldsymbol{L}(m-i)} .
$$

Then we have the following cases.

- When $i=0$, the quantity on the righthand side is

$$
\widetilde{X}_{\boldsymbol{L}(m)}^{*} \boldsymbol{M}_{\boldsymbol{L}(m), \boldsymbol{L}(m)}=1=\left|O_{\boldsymbol{L}(m)}\right| .
$$

- When $i=1$, the quantity on the righthand side is

$$
\tilde{X}_{\boldsymbol{L}(m)}^{*} \boldsymbol{M}_{\boldsymbol{L}(m), \boldsymbol{L}(m-1)}=1(m L)=\left|O_{\boldsymbol{L}(m-1)}\right| .
$$


- When $i \equiv 0(\bmod 4)$ and $i \neq 0$, the quantity on the righthand side is

$$
\begin{aligned}
\widetilde{X}_{\boldsymbol{L}(m-i+1)}^{*} & \boldsymbol{M}_{\boldsymbol{L}(m-i+1), \boldsymbol{L}(m-i)}+\widetilde{X}_{\boldsymbol{L}(m-i)}^{*} \boldsymbol{M}_{\boldsymbol{L}(m-i), \boldsymbol{L}(m-i)} \\
= & \left(\frac{1}{i-1}\left(\begin{array}{c}
m \\
i-2
\end{array}\right) L^{i-2}\right) L(m-i+1) \\
& +\frac{1}{i}\left(\left(\begin{array}{c}
m \\
i-1
\end{array}\right) L^{i-1}-\frac{1}{i-1}\left(\begin{array}{c}
m \\
i-2
\end{array}\right) L^{i-2}\right) \\
\leq & \left(\frac{m-i+1}{i-1}\left(\begin{array}{c}
m \\
i-2
\end{array}\right)+\frac{1}{i}\left(\begin{array}{c}
m \\
i-1
\end{array}\right)\right) L^{i-1} \\
\leq & \left(\begin{array}{c}
m \\
i
\end{array}\right) L^{i}=\left|O_{\boldsymbol{L}(m-i)}\right| .
\end{aligned}
$$

- When $i \equiv 1(\bmod 4)$ and $i \neq 1$, the quantity on the righthand side is

$$
\begin{aligned}
& \tilde{X}_{\boldsymbol{L}(m-i+1)}^{*} \boldsymbol{M}_{\boldsymbol{L}(m-i+1), \boldsymbol{L}(m-i)} \\
& \quad=\frac{1}{i-1}\left(\left(\begin{array}{c}
m \\
i-2
\end{array}\right) L^{i-2}-\frac{1}{i-2}\left(\begin{array}{c}
m \\
i-3
\end{array}\right) L^{i-3}\right) L(m-i+1) \\
& \leq \frac{m-i+1}{i-1}\left(\begin{array}{c}
m \\
i-2
\end{array}\right) L^{i-1} \\
& \leq\left(\begin{array}{c}
m \\
i
\end{array}\right) L^{i}=\left|O_{\boldsymbol{L}(m-i)}\right| .
\end{aligned}
$$

- When $i \equiv 2(\bmod 4)$, the quantity on the righthand side is

$$
\widetilde{X}_{\boldsymbol{L}(m-i-1)}^{*} \boldsymbol{M}_{\boldsymbol{L}(m-i-1), \boldsymbol{L}(m-i)}=\left(\frac{1}{i}\left(\begin{array}{c}
m \\
i
\end{array}\right)\right) i=\left|O_{\mathbf{L}(m-i)}\right| .
$$

- When $i \equiv 3(\bmod 4)$, the quantity on the righthand side is

$$
\begin{aligned}
& \widetilde{X}_{\boldsymbol{L}(m-i)}^{*} \boldsymbol{M}_{\boldsymbol{L}(m-i), \boldsymbol{L}(m-i)}+\widetilde{X}_{\boldsymbol{L}(m-i-1)}^{*} \boldsymbol{M}_{\boldsymbol{L}(m-i-1), \boldsymbol{L}(m-i)} \\
& =\frac{1}{i}\left(\begin{array}{c}
m \\
i-1
\end{array}\right) L^{i-1}+\frac{1}{i+1}\left(\left(\begin{array}{c}
m \\
i
\end{array}\right) L^{i}-\frac{1}{i}\left(\begin{array}{c}
m \\
i-1
\end{array}\right) L^{i-1}\right)(i+1) \\
& =\left(\begin{array}{c}
m \\
i
\end{array}\right) L^{i}=\left|O_{\boldsymbol{L}(m-i)}\right| .
\end{aligned}
$$

Next, we look at the column indexed by indexed by $\boldsymbol{K}(m-i)$ for $1 \leq i \leq m$. Recall that $\left|O_{\boldsymbol{K}(m-i)}\right|=m\left(\begin{array}{c}m-1 \\ i-1\end{array}\right) L^{i-1}\left(\begin{array}{c}L \\ 2\end{array}\right)$. Since most entries of $M$ are zero, we have that

$$
\begin{aligned}
\sum_{\boldsymbol{v} \in \mathcal{P}_{\text {row }}} & \widetilde{X}_{\boldsymbol{v}}^{*} \boldsymbol{M}_{\boldsymbol{v}, \boldsymbol{K}(m-i)} \\
& =\widetilde{X}_{\boldsymbol{L}(m-i)}^{*} \boldsymbol{M}_{\boldsymbol{L}(m-i), \boldsymbol{K}(m-i)}=\widetilde{X}_{\boldsymbol{L}(m-i)}^{*}(L-1) i .
\end{aligned}
$$

Since $\widetilde{X}_{\boldsymbol{L}(m-i)}^{*}$ is nonzero only in certain instances, we consider the following.

- When $i=1$, the quantity on the righthand side is

$$
L-1<m\left(\begin{array}{l}
L \\
2
\end{array}\right)=\left|O_{K(m-1)}\right| .
$$

- When $i \equiv 0(\bmod 4)$ and $i \neq 0$, the quantity on the righthand side is

$$
\begin{gathered}
\frac{1}{i}\left(\left(\begin{array}{c}
m \\
i-1
\end{array}\right) L^{i-1}-\frac{1}{i-1}\left(\begin{array}{c}
m \\
i-2
\end{array}\right) L^{i-2}\right)(L-1) i \\
\leq\left(\frac{1}{i}\left(\begin{array}{c}
m \\
i-1
\end{array}\right) L^{i-1}\right)(L-1) i \\
\leq m\left(\begin{array}{c}
m-1 \\
i-1
\end{array}\right) L^{i-1}\left(\begin{array}{c}
L \\
2
\end{array}\right)=\left|O_{K(m-i)}\right| .
\end{gathered}
$$


- When $i \equiv 3(\bmod 4)$, the quantity on the righthand side is

$$
\begin{aligned}
& \left(\frac{1}{i}\left(\begin{array}{c}
m \\
i-1
\end{array}\right) L^{i-1}\right)(L-1) i \\
& \quad \leq m\left(\begin{array}{c}
m-1 \\
i-1
\end{array}\right) L^{i-1}\left(\begin{array}{l}
L \\
2
\end{array}\right)=\left|O_{\boldsymbol{K}(m-i)}\right|
\end{aligned}
$$

Finally, we verify property (iii) for Definition 9 Indeed, we have that

$$
\begin{aligned}
& \sum_{\boldsymbol{u} \in \mathcal{P}_{\mathrm{col}}}\left|O_{\boldsymbol{u}}\right| \widetilde{Y}_{u}^{*} \\
= & \frac{m L}{m L}+\sum_{i=0}^{\lfloor m / 4\rfloor-1} \frac{1}{4 i+4}\left(\left(\begin{array}{c}
m \\
4 i+2
\end{array}\right) L^{4 i+2}+\left(\begin{array}{c}
m \\
4 i+3
\end{array}\right) L^{4 i+3}\right) \\
= & 1+\sum_{i=0}^{\lfloor m / 4\rfloor-1} \frac{1}{4 i+4}\left(\left(\begin{array}{c}
m \\
4 i+2
\end{array}\right) L^{4 i+2}+\left(\begin{array}{c}
m \\
4 i+3
\end{array}\right) L^{4 i+3}\right)
\end{aligned}
$$

and

$$
\begin{aligned}
& \sum_{\boldsymbol{u} \in \mathcal{P}_{\text {row }}} \tilde{X}_{\boldsymbol{u}}^{*} \\
& =1+\sum_{i=0}^{\lfloor m / 4\rfloor-1} \frac{1}{4 i+3}\left(\begin{array}{c}
m \\
4 i+2
\end{array}\right) L^{4 i+2}+ \\
& \frac{1}{4 i+4}\left(\left(\begin{array}{c}
m \\
4 i+3
\end{array}\right) L^{4 i+3}-\frac{1}{4 i+3}\left(\begin{array}{c}
m \\
4 i+2
\end{array}\right) L^{4 i+2}\right) \\
& =1+\sum_{i=0}^{\lfloor m / 4\rfloor-1} \frac{1}{4 i+4}\left(\left(\begin{array}{c}
m \\
4 i+2
\end{array}\right) L^{4 i+2}+\left(\begin{array}{c}
m \\
4 i+3
\end{array}\right) L^{4 i+3}\right) .
\end{aligned}
$$

Therefore, equality holds and we have verified the conditions for $\left(\widetilde{\boldsymbol{Y}}^{*}, \widetilde{\boldsymbol{X}}^{*}\right)$ to be an optimality certificate.

\section{REFERENCES}

[1] A. Tandon, M. Motani, and L. R. Varshney, "Subblock-constrained codes for real-time simultaneous energy and information transfer," IEEE Trans. Inform. Theory, vol. 62, no. 7, pp. 4212-4227, Jul. 2016.

[2] S. Zhao, "A serial concatenation-based coding scheme for dimmable visible light communication systems," IEEE Commun. Lett., vol. 20, no. 10, pp. 1951-1954, Oct. 2016.

[3] Y. M. Chee, Z. Cherif, J.-L. Danger, S. Guilley, H. M. Kiah, J.-L. Kim, P. Sole, and X. Zhang, "Multiply constant-weight codes and the reliability of loop physically unclonable functions," IEEE Trans. Inform. Theory, vol. 60, no. 11, pp. 7026-7034, Nov. 2014.

[4] Y. M. Chee, H. M. Kiah, and P. Purkayastha, "Matrix codes and multitone frequency shift keying for power line communications," in Proc. 2013 IEEE Int. Symp. Inf. Theory, Jul. 2013, pp. 2870-2874.

[5] A. Tandon, M. Motani, and L. R. Varshney, "Real-time simultaneous energy and information transfer," in Proc. 2015 IEEE Int. Symp. Inf. Theory, Jun. 2015, pp. $1124-1128$.

[6] A. Tandon, H. M. Kiah, and M. Motani, "Bounds on the size and asymptotic rate of subblock-constrained codes," IEEE Trans. Inform. Theory, vol. 64, no. 10, pp. 6604-6619, Oct. 2018.

[7] A. Fazeli, A. Vardy, and E. Yaakobi, "Generalized sphere packing bound," IEEE Trans. Inform. Theory, vol. 61, no. 5, pp. 2313-2334, May 2015.

[8] A. A. Kulkarni and N. Kiyavash, "Nonasymptotic upper bounds for deletion correcting codes," IEEE Trans. Inform. Theory, vol. 59, no. 8, pp. 5115-5130, 2013.

[9] D. Cullina and N. Kiyavash, "Generalized sphere-packing bounds on the size of codes for combinatorial channels," IEEE Trans. Inf. Theory, vol. 62, no. 8, pp. 4454-4465, 2016.

[10] C. Freiman, "Upper bounds for fixed-weight codes of specified minimum distance (Corresp.)," IEEE Trans. Inform. Theory, vol. IT-10, no. 3, pp. 246-248, 1964.

[11] E. Berger, "Some additional upper bounds for fixed-weight codes of specified minimum distance," IEEE Trans. Inform. Theory, vol. IT-13, no. 2, pp. 307-308, 1967.

[12] F. Margot, "Exploiting orbits in symmetric ilp," Mathematical Programming, vol. 98, no. 1-3, pp. 3-21, 2003.

[13] R. Bödi and K. Herr, "Symmetries in linear and integer programs," arXiv preprint arXiv:0908.3329, 2009.

[14] V. Chvatal, Linear programming. Macmillan, 1983. 